\title{
SIMULATION OF FLIGHT STATES FOR FLEXIBLE FALLING PLATES BY THE IMMERSED BOUNDARY METHOD
}

\author{
EDWIN M. LAU ${ }^{1}$ AND WEI-XI HUANG ${ }^{2}$ \\ ${ }^{1}$ Department of Aeronautical Engineering, Chaoyang University of Technology \\ Taichung, Taiwan, 41349 \\ edwin@cyut.edu.tw \\ ${ }^{2}$ AML, Department of Engineering Mechanics, Tsinghua University \\ Beijing 100084, P.R. China \\ hwx@tsinghua.edu.cn
}

Key words: Flexible plate, Flight state, Immersed boundary method

\begin{abstract}
The falling of a plate is a simply posed problem that offers complex fluid-structure interactions. It also gives rise to different flow structures in relation to specific flight patterns of a plate such as autorotating tumble, side-to-side flutter, or constant abrupt turning in a chaotic manner depending on the Reynolds number and the non-dimensional moment of inertia In actual falling flights such as tree leaves and organisms, the bodies possess flexibility. Here, direct numerical simulation is used to portray the free fall of a flexible plate. The Navier-Stokes equations are solved via the upper-lower decomposition method. The effect of the flow on the solid plate is transferred through the immersed boundary method. A strictly spatially dependent stencil of the IB is adapted to provide a solution to the fourth-order beam equation via the pent-diagonal matrix algorithm. Comparing to the conventional method that couples with the temporal domain, this enables stability of solution through a range of bending rigidity $k$ from $k=50$ to $k=0.01$. A plate with high stiffness resembles to a plate of non-flexible solution. The tumbling flight trajectory is found to change as a plate is more flexible, or as $k$ is decreased. Further decrease in $k$ result in changes flight states.
\end{abstract}

\section{INTRODUCTION}

The smooth, swift motion of a falling leaf is the result of complex fluid-structure interactions (FSI). The earliest works began from the interest of a freely falling object that appear to be influenced by forces other that gravity. Maxwell [1] deduced the first theoretical explanation via the flow resistance. Willmarth et al. and Field et al. [2,3] distinguished the tumbling, chaotic, and fluttering flight states defined by the Reynolds number, $R e$, and the non-dimensional moment of inertia of the plate, $I^{*}$. Natural passive flights are often thin and flexible. They deform under their own weight as well as fluid forces. The effect of flexibility in fluid interactions has been extensively studied in biological organisms and micro aerial vehicles at relatively low Reynolds numbers $\left(\operatorname{Re}<10^{3}\right)$.

There are different effects for different forms of flexible flight of flexible plates [4]. One of the efficient way of investigating this problem is through numerical methods. The use of direct numerical simulation (DNS) with immersed boundary (IB) method yields a two-way coupled interaction for which this 
(a)

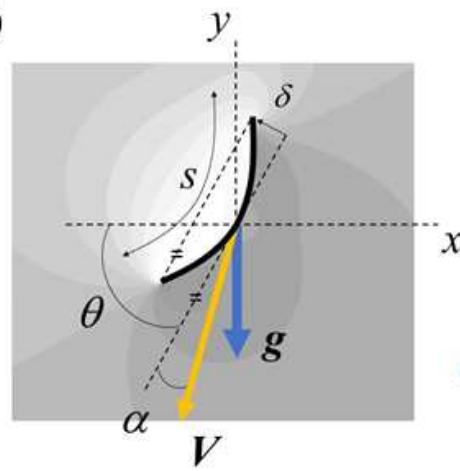

(b)

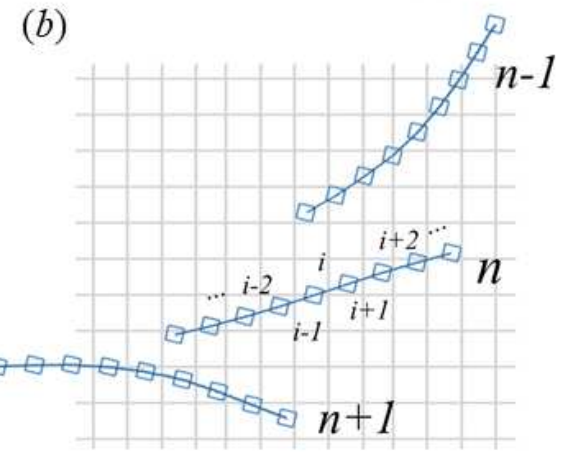

Figure 1: (a) Global Cartesian reference frame and nomenclature: $s$ as arclength, $V$ as velocity, $g$ as gravity, $x$ and $y$ as horizontal and vertical coordinates, $\alpha$ as angle of attack between the chord and $V, \delta$ as deflection, $\theta$ as pitch angle between the chord and the $x$-axis, respectively. (b) Schematic of the Lagrangian coordinate system for a flexible plate at three time instances: $n$ as time step; $i$ as Lagrangian point number.

problem is required [5]. To implement flexibility, Zhu et al. [6] used an stencil based on different and temporal and spatial IB points. The flexible motion is thus, determined and also confined by the previous space and time steps. Within the fundamental problems of hinged or prescribed flexible motion, this method is very effective. However, for a plate falling in passive flight, the motion is not determined beforehand. Both the flexibility and the velocity have confined values such that numerical stability issues may result in large fluctu simulations of different ort

This study introduces a fo to resolve the bending of is enhanced, and the rang ation of nonphysical forc
the plate throughout its pa
of $k$ to depict various for
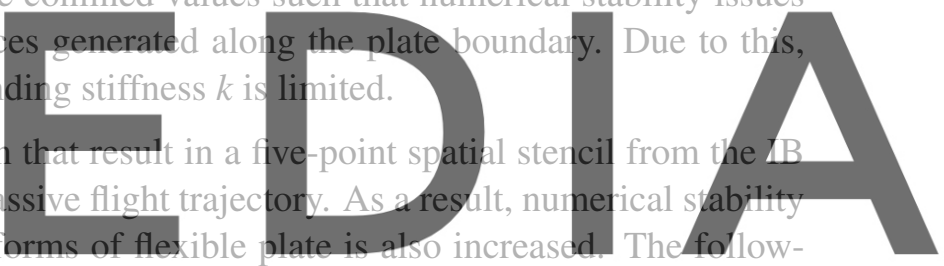

ing first introduce the derivation of the stencil towards the equations of motions. Then the simulated

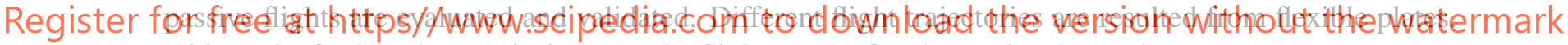
Ultimately, further changes in $k$ causes the flight states of a plate to be changed.

\section{METHOD}

The free fall of the plate is considered to be in a two-dimensional, viscous, incompressible fluid. The flow is derived from the Navier-Stokes equations, the plate is by the equation of motion, and their coupling is through the IB method [5].

Following the schematic and nomenclature in figure 1(a), the plate itself has a chord length $l$. The density of solid $\rho_{s}$ is higher than that of fluid $\rho_{f}$, such that it falls under the field of gravity $g$ (and $g=|\boldsymbol{g}|$ ) inside the fluid of viscosity $\mu$. While the IB is a one-dimensional line, the thickness $h$ of the plate is assumed to be negligible. When the fall is initiated, the plate generates a flow velocity field $\boldsymbol{u}$ $=\left(u_{x}, u_{y}\right)$ in the Eulerian domain. The convention of using $\rho_{f}, l$, and the reference descent velocity $V_{\infty}=$ $\left[2\left(\rho_{s} / \rho_{f}-1\right) g h\right]^{1 / 2}$ as the characteristic parameters are followed [7]. The subsequent dimensionless parameters are: $l / V_{\infty}$ for time $t, \rho_{f} V_{\infty}{ }^{2}$ for pressure $p, \rho_{f} V_{\infty}{ }^{2} / l$ for Eulerian force $f, \rho_{f} V_{\infty}{ }^{2} l$ for tension force $T, \rho_{f} V_{\infty}{ }^{2} l^{3}$ for bending rigidity $k$, and $\rho_{f} V_{\infty}{ }^{2}$ for Lagrangian force $\boldsymbol{F}$. Further defining the density ratio as $\rho^{*}=\rho_{s} / \rho_{f}$, and the Reynolds number as $R e=\rho_{f} l V_{\infty} / \mu$, the N-S equation (1), the continuity 
equation (2), and the equation of motion of flexible plate (3) are written in non-dimensional forms, i.e.

$$
\begin{gathered}
\frac{\partial u}{\partial t}+u \cdot \nabla u=-\nabla p+R e^{-1} \nabla^{2} u+f \\
\nabla \cdot u=0 \\
\rho \frac{\partial^{2} X}{\partial t^{2}}=\frac{\partial}{\partial s}\left(T \frac{\partial X}{\partial s}\right)-\frac{\partial^{2}}{\partial s^{2}}\left(k \frac{\partial^{2} X}{\partial s^{2}}\right)+\rho \frac{g}{g}-F
\end{gathered}
$$

Here, $\boldsymbol{F}=\left(F_{x}, F_{y}\right)$ and $\boldsymbol{f}=\left(f_{x}, f_{y}\right)$ are interaction forces between the solid and the fluid, respectively. The symbol $\rho=\left(\rho^{*}-1\right) h^{*}$ further simplifies the form of equation (3) with $h^{*}=h / l$. The non-dimensionalised moment of inertia is approximated as $I^{*}=\rho^{*} h^{*}$.

In the IB method, the interaction between the Lagrangian points and the adjacent fluid particle is provided through a feedback. Interpolation from the Eulerian fluid velocity $u=(u, v)$ to the Lagrangian points is done yia a smoothed delta function [?, 9]:

$$
U_{I B}(s, t)=\int_{\text {domain }} u(x, t) \delta(X(s, t)-x) d x
$$

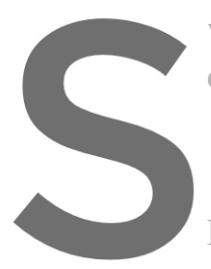

where $X=(X, Y)$ denotes the Lagrangian coordina
calculated by spreading the Lagrangian force to its
For clarity, in the IB formulation ( $(4)$ and (5), the 1

the upper case ones represent the Lagrangian variables.

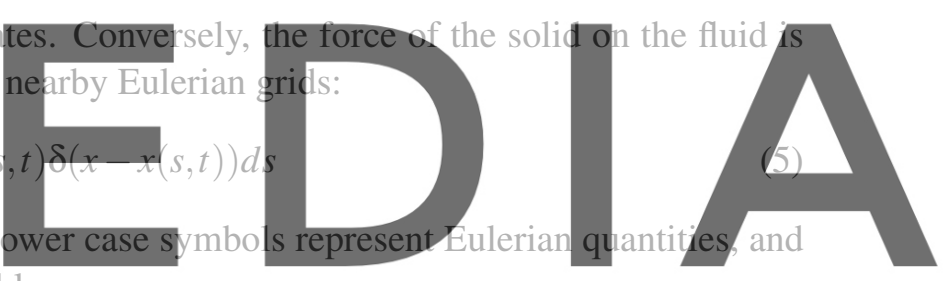

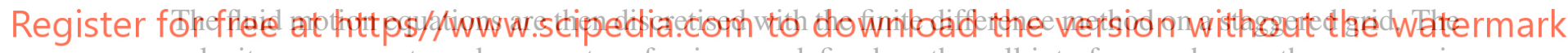

velocity components and momentum forcing are defined on the cell interfaces, whereas the pressure is

applied at the centre of cells. The decoupling of the velocity and pressure is achieved by the upper/lower

block decomposition through approximate factorization. The fractional step method is used for temporal advancement, and both the convective and viscous terms are discretised via the Crank-Nicholson scheme, preserving the second-order temporal accuracy [10]. The pressure Poisson equation is solved via fast Fourier transform, which is then used to correct the velocity field to satisfy the continuity equation in completing the fractional steps. The coupling between the fluid and the solid is abridged via the no-slip condition that is enforced by the IB method.

The plate is considered as inextensible in the chordwise direction. While it is possible to numerically confine the extension of the plate very close to zero by imposing a sufficiently large stretching coefficient [6], it would require a small computational time step to ensure the numerical stability. Instead, the geometric inextensible condition is imposed, i.e. $(\partial X / \partial s) \cdot(\partial X / \partial s)=1[13,5]$.

In considering the plate's flexibility, a five-point IB stencil is used (labelled $i$ in figure 1b) to solve the motion equation (3) via the pent-diagonal matrix algorithm with the following boundary conditions at the free ends of the plate at $s=0$ and $s=l[12]$ : 

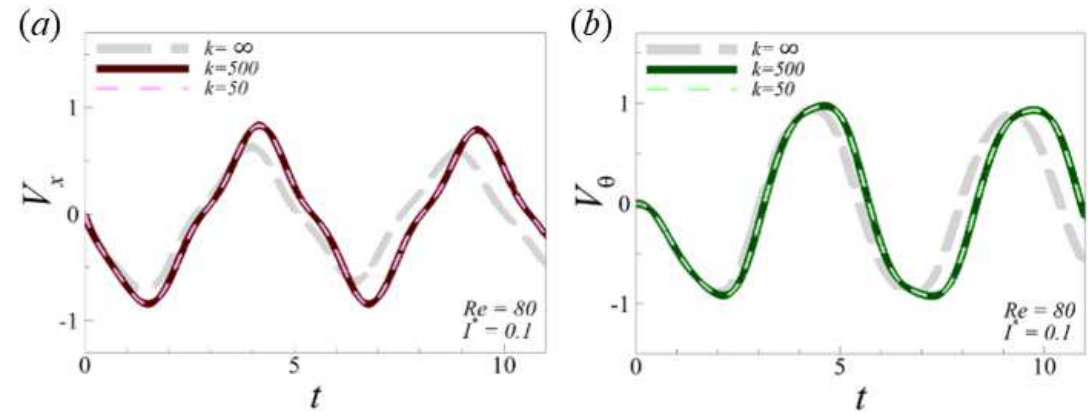

Figure 2: Time histories of fluttering plates at $R e=80$ and $I^{*}=0.1$. The current flexible solution at $k=50$ and $k=500$ are compared with a non-flexible solution [12] denoted as $k=\infty$, in terms of: (a) lateral velocity; (b) angular velocity.

$$
T=0, \quad \frac{\partial^{2} X}{\partial s^{2}}=(0,0) \quad \frac{\partial^{3} X}{\partial s^{3}}=(0,0)
$$

\section{RESULTS}

3.1 Performance

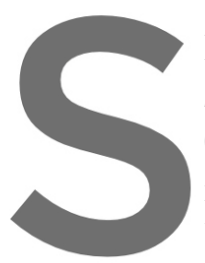

In performing each simulation, the plate begins to fall from rest. The bending rigidity is
$k<50$. The Reynolds number is varied in $\mathrm{range}$ of $50<R e<250$; and the moment of ine
$0.1<I^{*}<1.0$. This fourth order beam $\mathrm{B}$ method is validated by considering plates' flutt
in different $k$ values and methods, as depicted in figure 2 (a) \& (b). The laterd and angul
$V_{x}$ and $V_{\theta}$, are compared at $k=50$ and $k=500 \mathrm{w}$ ith respect to the formulation of a non-

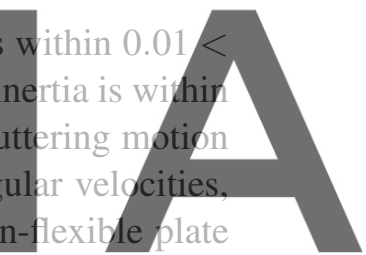

solution, labelled as $k=\infty$. $V_{x}$ provides a comparison of flight envelopes, while $V_{\theta}$ provides a comparison

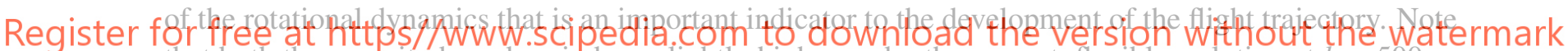
that both the magnitude and period are slightly higher under the current, flexible, solution at $k=500$ as compared $k=\infty$. The difference may be due to the different governing equations used.

Furthermore, figure 3 shows the results between the non-flexible solution (a), and the current fourth order beam solution in (b) set to be very rigid. It can be seen that, throughout the fluttering turn, the plate at $k=50$ has no deformation. Important features such as leading edge and trailing edge vorticities resembles closely to the non-flexible solution.

\subsection{Effect of flexibility on trajectories}

A plate of $k=0.01$ undergoes a stable tumbling trajectory in figure 4. It is very little different from conventional non-flexible trajectories [7]. The sequences of snapshots from figure 4(b) to (d) depict the two critical instances - turning and gliding. It shows that, within different vorticity and velocity fields, there may be small deformations of the plate as in (b), or large deformations as in (d).

The plate in figure 5, also in tumbling flight at the same $k$, appears to have less tendency rotate. It thereby generates a unique, aperiodic tumbling trajectory not found in previous passive flight studies. There are 

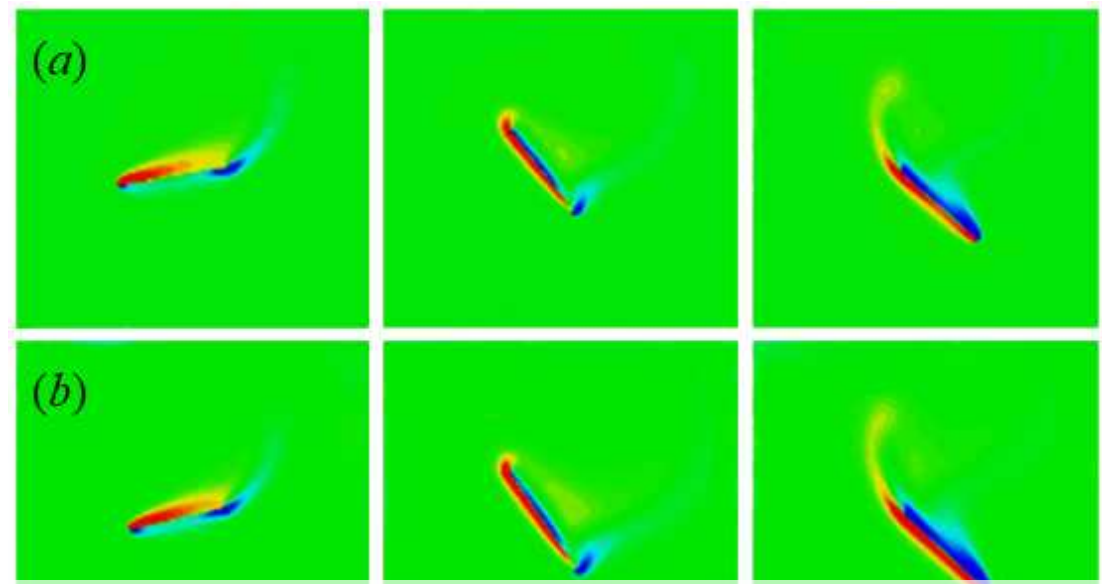

Figure 3: Vorticity fields of a falling plate at $R e=80$ and $I^{*}=0.1$ in three snapshots. (a) Solved via a non-flexible solution [12], denoted as $k=\infty$. (b) Solved with the current flexible solutions with $k=50$.

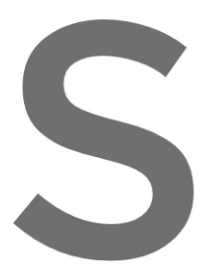

(a)
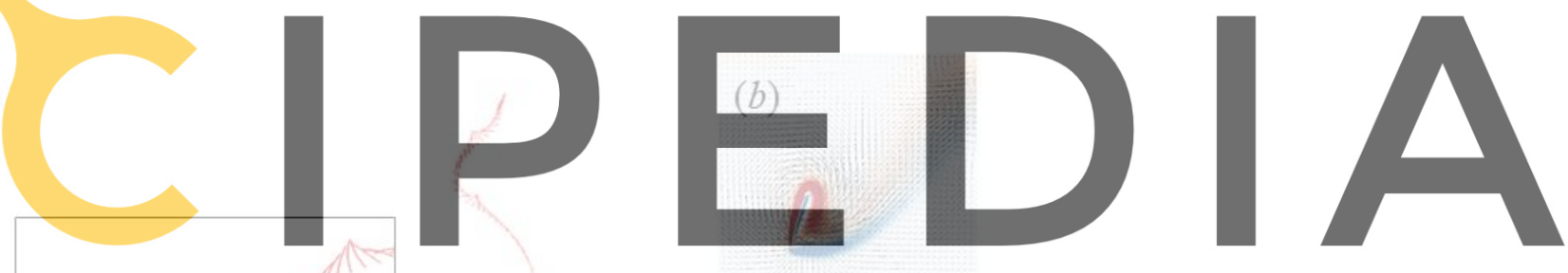

Register for free at https//www.scipedia.com to download the version without the watermark
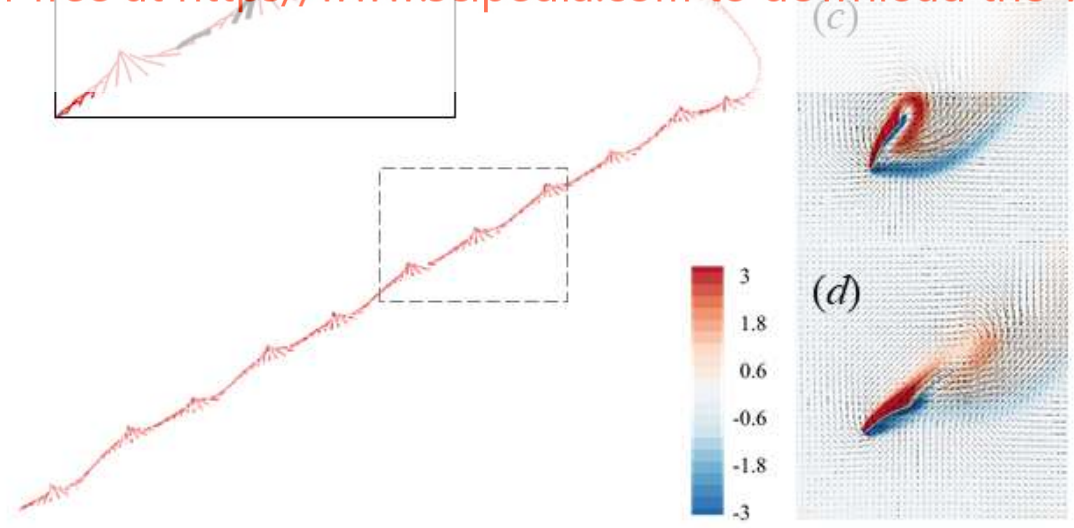

Figure 4: (a) Tumbling flight path of case $I^{*}=1.0, R e=100$ and $k=0.01$ with instants of (b, c, d) velocity and vorticity fields. 
(a)

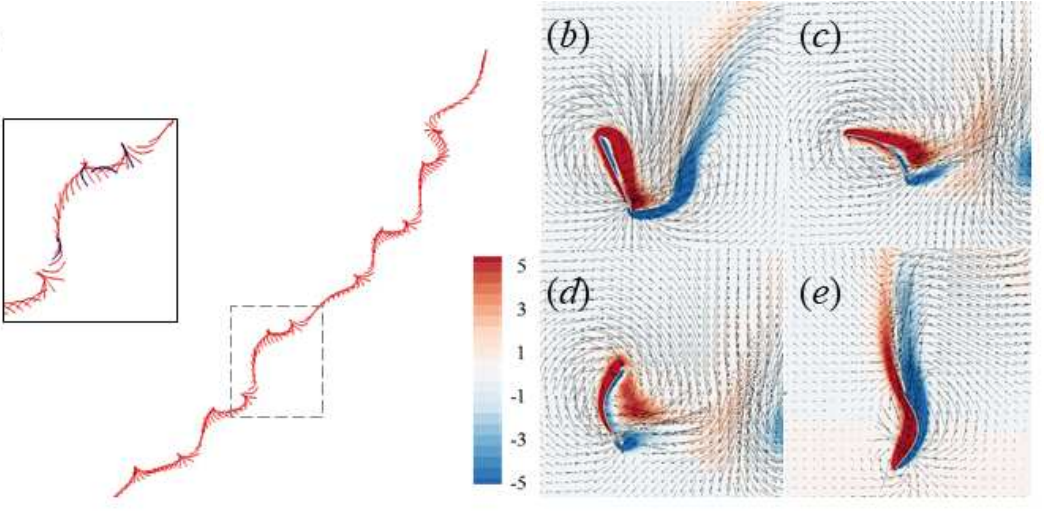

Figure 5: (a) Tumbling flight path of case $I^{*}=0.70, R e=200$ and $k=0.01$ with instants of (b, c, d, e) velocity and vorticity fields.

large degree of plate deformation shown in the vorticity-velocity fields of figure 5(c-d). This suggests that the degree of bending affects the flight path of the plate..

\subsection{Effect of flexibility on flight states}

Given the changes in FSI, further changes in $k$ eventually yield changes in the flight state of a plate altogether. With the numerical method enabling the changes in $k$ from 50 to 0.001 , figure 6 depicts, for a constant $R e$ and $I^{*}$, the change in flight states. chaotic flight in in figure more likely that a plate wi

4 CONCLUSION
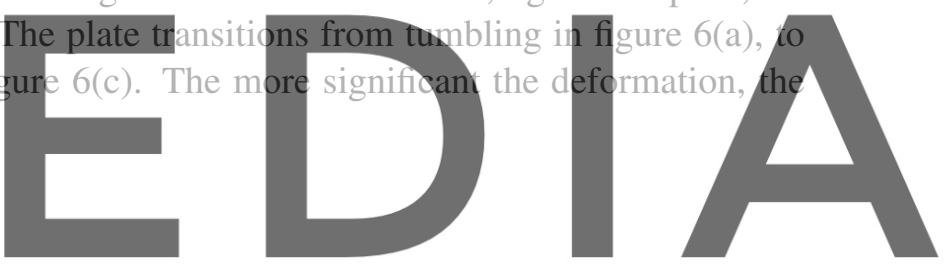

With the introduction of a five point stencil, the corresponding numerical discretisation of the equation of

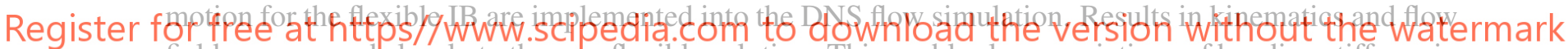
fields correspond closely to the non-flexible solution. This enables large variations of bending stiffness in

$O\left(10^{4}\right)$, for a problem that previously encountered numerical stability. It is found that plate trajectories are changed as bending stiffness is decreased. Large decrease in $k$ result in changes in flight states. The formulation of flexible plates can be applied to observe changes in passive flight for plates in larger range of Reynolds number and moment of inertia.

WCCM-ECCOMAS2020@ cimne.upc.edu

\section{REFERENCES}

[1] Maxwell, J.C. Draft of paper on the descent of a body in a resisting medium. Cambridge University Press, (1852).

[2] Willmarth, William W. and Hawk, Norman E. and Harvey, Robert L. Steady and unsteady motions and wakes of freely falling disks. Physics of Fluids (1964) 7,2:197-208.

[3] Field, Stuart B. and Klaus, M. and Moore, M. G. Chaotic dynamics of falling disks. Nature (1997) 388:252-254. 


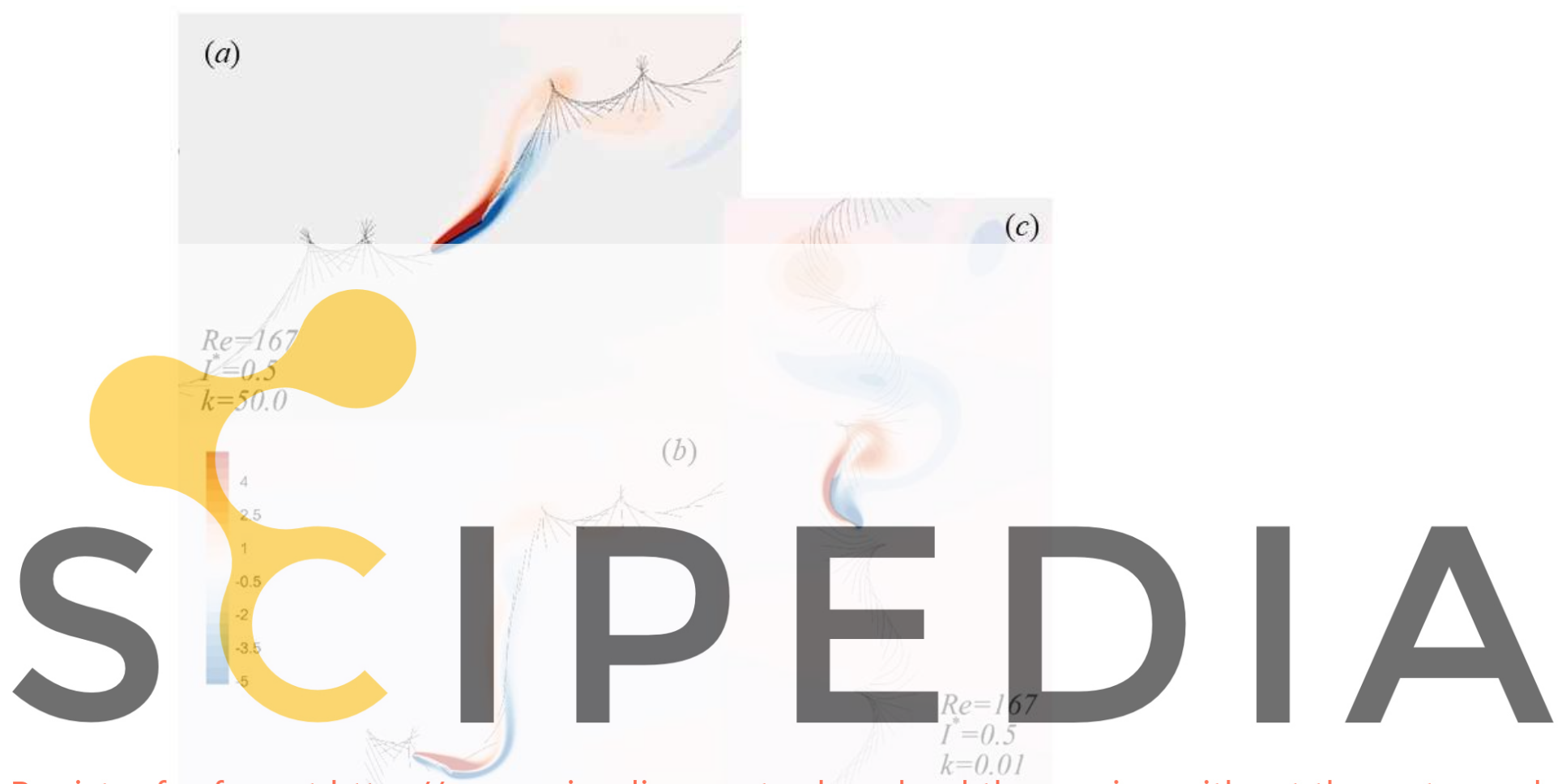

Register for free at https//www.scipedia.com to download the version without the watermark

$$
\begin{aligned}
& R e=167 \\
& I^{*}=0.5 \\
& k=0.05
\end{aligned}
$$

Figure 6: Trajectories and instantaneous vorticity field of plates in different flight states at $R e=167$ and $I^{*}=0.5$ with various bending stiffness: (a) tumbling at $k=50$, (b) chaotic at $k=0.05$ and (c) fluttering at $k=0.01$. 
[4] Shyy, W. and Aono, H. and Chimakurthi, S. K. and Trizila, P. and Kang, C. K. and Cesnik, C. E. S. and Liu, H. Recent progress in flapping wing aerodynamics and aeroelasticity. Progress in Aerospace Sciences (2010) 46,7:284-327.

[5] Huang, Wei-Xi and Shin, Soo Jai and Sung, Hyung Jin. Simulation of flexible filaments in a uniform flow by the immersed boundary method. Journal of Computational Physics (2007) 226,2:2206-2228.

[6] Zhu, Luoding and Peskin, Charles S. Simulation of a Flapping Flexible Filament in a Flowing Soap Film by the Immersed Boundary Method. Journal of Computational Physics (2002) 179,2:452-468.

[7] Andersen, A. and Pesavento, U. and Wang, Z. Jane. Unsteady aerodynamics of fluttering and tumbling plates. Journal of Fluid Mechanics (2005) 541:65-90.

[8] Zhu, Luoding. Simulation of an inhomogeneous elastic filament falling in a flowing viscous fluid. Physics of Fluids (2007) 19:01113.

[9] Peskin, Charles S. The immersed boundary method. Acta Numerica (2002) 11.

[10] Kim, Yongsam and Peskin, Charles S. Penalty immersed boundary method for an elastic boundary with mass. Physics of Fluids (2007) 19, 5:053103.

[11] Andersen, A. and Pesavento, U. and Wang, Z. Jane Tornberg, Anna-Karin and Shelley, Michael J. Simulating the dynamics and interactions of flexible fibers in Stokes flows. Journal of Computational Physics (2004) 196,1:8-40.

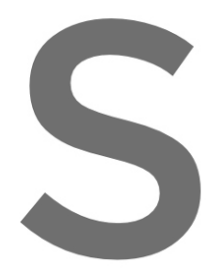

[12] Lau, Edwin M. and Huang, Wei-Xi and Xu, falling to tumbling

13] Andersen, A. and Simulating the dy tional Physics (2004) 196,1:8-40.

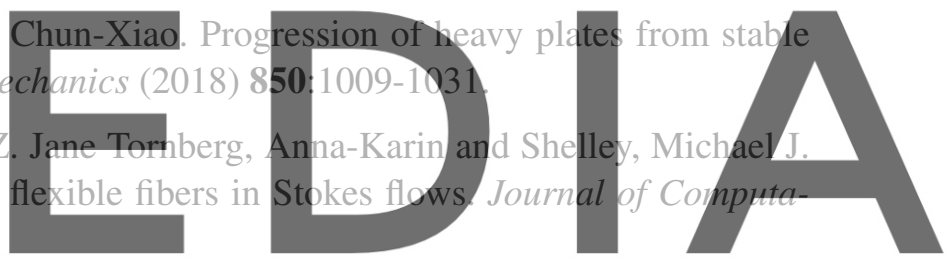

